\title{
A ReAlist Model of CoMmunication APPLICATIONS FOR INFORMATIONAL TECHNOLOGY AND ARTIFICIAL COGNITIVE SYSTEMS
}

\author{
Monica R. Kimmel \\ Development of communicational strategies, Collibri NGO, Gothenburg, Sweden
}

\begin{abstract}
The structure of the Shannon-Weaver model of communication (1948) has been the point of departure for the coming communication models that ever since have functioned as a base for developing systems and strategies within all the fields that depend on informational transfer. Attempts to develop the model have not affected its basic structure, or the use of the central concept of information. As it will be argued in this paper, this presents a number of limitations to the development of informational technology and more functional artificial cognitive systems. After contextualizing and adapting a number of central concepts for any theory of communication, the author presents a model of communication that is viable as a foundation for the development of artificial cognitive systems used for functions like natural language-processing, object-recognition and machine-learning.
\end{abstract}

\section{KEYWORDS}

Quantification of Information, Intentionality, Intelligence, Object-Recognition, Machine-Learning.

\section{Purpose of the Model And its Methodological Limitations}

In Shannon's paper on informational transfer from 1948, he states from the outset that the "fundamental problem of communication is that of reproducing at one point either exactly or approximately a message selected at another point" [1]. This is the engineering problem. Shannon also delimits the functional range of the model to the technical aspect of the model, thus excluding the semantic dimension and the effectual dimension of the problem of reproducing "exactly or approximately" a message from point A to point B. The primary function of a communicational model is, according to this original view, to represent the reproduction of an informative content from one point to another. This process is by Shannon illustrated as in Figure 1. Shannon's exclusion of the semantic aspects of information is in turn inspired by Hartley's theory of the transmission of information that was published in 1927. Hartley aims at quantifying information and distinguishes in connection to this between the physical and psychological considerations [2]. These arduous attempts to quantify information without involving any interpretative factors have their background in the obscure nature of the human cognitive functions. In spite of the progress that neuropsychology and other related fields have made in mapping the human cognitive functions, very little is known about how, for instance, humans interpret visual data or process natural language. The lack of knowledge in these fields is an obstacle to quantifying the factors of importance for a complete account of informational transfer. If one cannot predict or formulate mathematically what interpretation a particular selected message will have, if the interpretation is dependent on the subjective preconditions of each 
individual, it is not appropriate to include the aspect of interpretation in a scientific methodological toolbox.

On the other hand, the foundation of objectivity is intersubjectivity; the validity of any scientific result depends on the possibility of testing and reproducing and communicating that result to other subjects in a comprehensible way so that they in turn can evaluate and reproduce those same results. Weaver links in 1949 the notion of information to Shannon's theory of entropy and ascribes language the character of statistical probability that by Shannon is described as a basic means of quantifying information [3]. Weaver noted in that same article that there may be a problem with reducing the notion of information to its technical aspect because "a theoretical analysis of the technical problem reveals that it overlaps the semantic and the effectiveness problems more than one might suspect". Nevertheless, Weaver concludes the subject by suggesting that "perhaps meaning may be shown to be analogous to one of the quantities on which the entropy of a thermodynamic ensemble depends." (1949, p. 15).

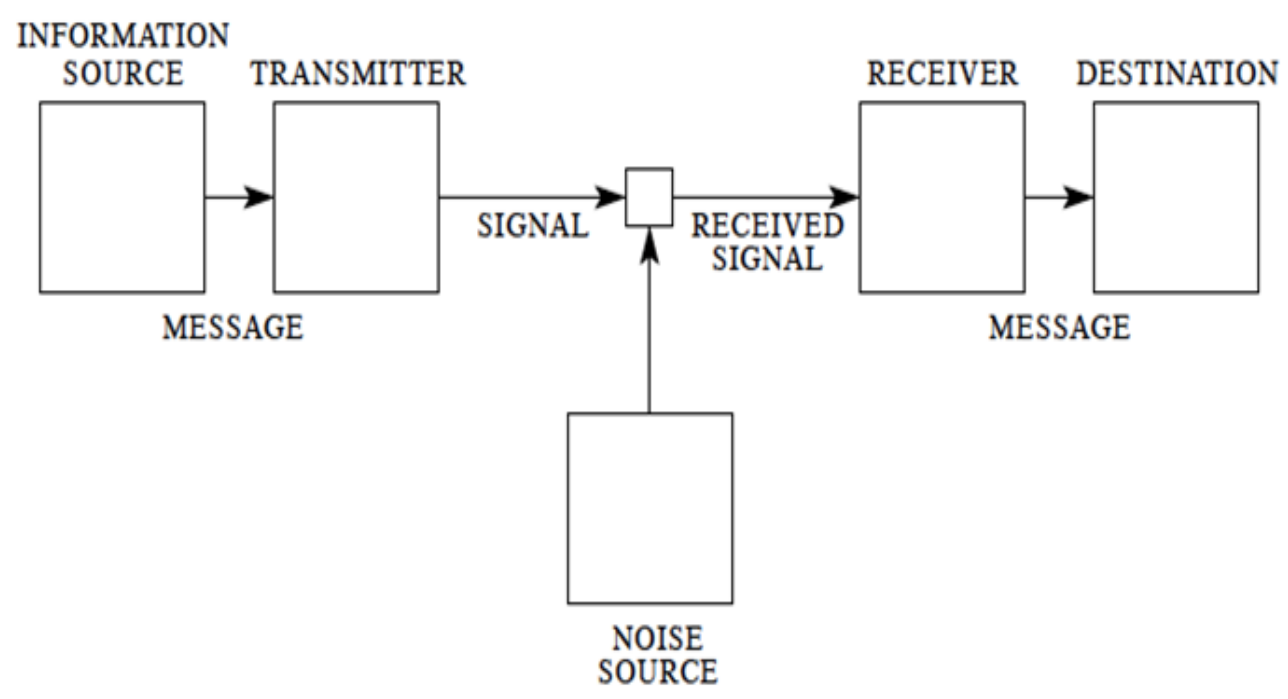

Figure 1. Shannon's general model of communication.

While Weaver drags semantics into the mathematics of information to solve the dilemma of meaningful informational transference, various more or less serious attempts have been made to put the aspect of meaning back into information [4]. For a detailed analysis of these attempts see Kimmel and Mutto (2019) [5]. The challenge of quantifying meaning is seldom addressed, and when it is, meaning is ascribed to the structural composition of data. In order to address quantification of meaning it is necessary to (1) be clear about how the central concepts surrounding questions of meaning are being applied, and (2) analyse in detail the structure of the preconditions of meaningfulness - which in this paper are identified as the human perceptual system. The human cognitive system is depending on perceptual input in order to have a content that it can process, just like processors in machines are dependent on input of data to have something to process. The difference is that the content that the human perceptual system abstracts from the environment is more determined by the structure of the cognitive system than, for instance, the input of data in a computer is determined by its operative system. This leads to several consequences for the Shannon-model (and the models that it has mothered), but two major aspects that imply difficulties for applications of the model are; that it messes with the generality of the model (not applicable to human communication), and that it distorts the notion of 'information' in ways that may be difficult to sort out. 
As the aim of this paper is to suggest a model of communication and a communication theory that can be applied to different actual communicational situations (generality), and be practical to use in the planning, analysis and troubleshooting of informational transfer, the tools that will be used come from two separate philosophical traditions that have often been considered to be incompatible; the analytical tradition and the phenomenological. (1) The conceptual analysis and working definitions are made with the aid of comparative and applicational analysis and are measured by their applicational (practical) and differentiating values. (2) For the structural analysis, especially since the main problem is how to quantify meaning, we again need a combination of tools from both the analytical and the phenomenological tradition. Frege's principles of compositionality and contextuality have contributed with the basics for understanding the minimal requirements for meaningfulness. From Husserl's analysis of perceptual experience the notions of 'hyletic data' (raw data), 'apperception' and 'setting' of objects as real, are borrowed in order to complete the Fregean notion of 'thought' (which is binary) and how 'thoughts' are being grasped (understood, made informative). A necessary concept to fully appreciate the contribution that Husserl makes to the theory of communication is intentionality, in this paper borrowed from Brentano.

Since both the conceptual and the structural analysis here are performed by contextualization and measured by their applicational and differentiating value, the most suitable counterpart of comparison is artificial cognitive systems. Artificial cognitive systems are constructed to mimic the ways humans learn, identify objects in their environment and process natural language. In areas like calculation, analysis and synthesis, the artificial cognitive systems have reached higher levels of speed and accuracy than human cognitive systems. In areas like machine learning, natural language processing and object identification, programmers are still struggling to achieve levels of speed and accuracy that can be compared to the human levels. There are also very good reasons to aim at achieving levels of speed and accuracy on these areas that exceed the human levels (or at least comparable to), since they have the potential to aid humans in various tasks or be applied in areas like assistive technology - for instance, for the visually and auditively impaired.

The questions we need to ask before we proceed is whether it is sufficient to artificially imitate the thinking human cognitive system, or if artificial cognitive systems can be (or need to be) developed to think for themselves. Turing's position (1950) on the matter of machine's capability of thinking is an illustration of this point. Turing believed that it is sufficient that machines can imitate the processes involved in thinking for us to say that machines "think". While confronting Lovelace's argument from 1842 that a computer cannot do something that it is not programmed to do, Turing expresses - with reference to Hartree's counterargument - an optimism concerning what future computational machines will be able to do [6]. We can consider the Turing-test the weaker requirement and the Lovelace-test the stronger. Initially, we have to keep two separate questions apart:

(A) How can we program a machine to mimic that which humans do when they think?

(B) How can we program machines to think for themselves?

And the more important question: is (A) or (B) necessary (or both) for the development of artificial cognitive systems that can be applied on areas like for instance assistive technology? To reconnect to the model of communication; if it turns out - as the author of this paper aims to show - that the Shannon-model does not represent the human communicational process accurately, and that the processes involved in object identification, language-processing and learning are not correctly (or not at all) represented by the communicational model, what alternatives are there to bring about (A) or (B) or both? 
However useful Shannon's theory of entropy has been for the development of artificial systems that learn, process natural language and recognize objects, we seem to have inherited the dilemma of quantifying meaning from Shannon's (and others) conception of information. The dilemma is this: if, as Shannon and others argue, the main problem of communication is to reproduce a selected message (informative content) at another point than the point of origin, then we need criteria according to which we can ascertain that the original message has been reproduced at another point. To construct this type of criteria, first we need a clear and well demarcated set of concepts.

\section{EXPLICATION OF SOME CONCEPTS RELATED TO INFORMATION}

In order to outline a model of communication that satisfies both the criteria of objectivity and of subjectivity - i.e. both the aspect of mathematical accountability/predictability and the aspect of meaning - it is necessary to be clear about the use of the central concepts that surround the subject of communication. The concept "communication" is usually defined as an exchange of information. "Information" on the other hand is defined both as data and as obtained knowledge. "Knowledge" being tightly knitted with learning, is usually described in connection to awareness, experience and understanding. These concepts being perhaps the most debated in the history of philosophy, are central to the understanding of what types of processes that are a part of a communicational act, as well as to the development of a model of communication that correctly represents the actual communicational acts that enable social life, science, knowledge acquisition and progress through history by inheritance of the knowledge of previous generations.

Communication is in this context initially considered to be an exchange of units (symbolic, linguistic) of content that can be interpreted into information of some kind. It is thus not sufficient to say that some symbol or some code has been transmitted from A to B - however successfully so - if the symbol or code is not structured in a way that can be made sense of or interpreted into meaningful bits of information. To the question of the conditions of some set of symbols or data to be interpretable or meaningful, to what type of structure that is the minimal requirement, we need a detailed discussion of both the logico-mathematical and experiential aspects of meaningfulness. These discussions will dominate section 3 of this paper. The mentioned necessary criteria for communication (that an organized structure is at the basis of the sense-making) is not sufficient though, for an act to be called communicative it is also necessary that the message is interpreted into some informative unit by a recipient of the message.

The concept of information will be used in similar ways: content of a selected set of symbols or code that cannot be made sense of is not informative, and content that can be made sense of but is never interpreted cannot be called information.

Information is not necessarily a part of a communication process or of a message of some sort. In this sense, for instance Capurro and Hjørland's initial description of information as "knowledge communicated" [7] is not appropriate as a definition of information. Information is always something composed by an interpreting agent, whether the interpretation is of a selected message from another source or from an object or phenomenon under investigation. So, first of all, information is not necessarily communicated. In some situations, one could say that information is "knowledge" - a concept to which we soon will have to relate - though if the definition of knowledge includes the condition of truth (as in the classical true, justified, belief) this description is also incorrect. That is because false information is still information, i.e. structure interpreted into meaning. Even false propositions can be interpreted into meaningful units and as such be informative. 
The consequences of this for the forthcoming construction of the realist model of communication are quite dramatic. For instance, information can no longer be said to "travel" via "channels" and if it is not dependent on "channels" to move from A to B, the "noise" that has such an important role in Shannon's model of communication can no longer have an impact on the information (see Figure 1. above). Bawden and Robinson revisit Shannon's notion of entropy and analyse the implications of the applications of the notion of entropy as a description of "missing information" [8]. In the context of this paper and the usage of the term "information", entropy becomes more like missing data or damaged code. Entropy as it is related to the mathematical concept of information is thus less relevant to the notion of information in this context, unless it can be explained in terms of interpretation of selected bits of code.

A system that is able to make sense of symbols and to interpret patterns into meaningful units needs to have some form of structure or program to do so. A preset pattern according to which symbols are organized and turned into informational units will in this context be termed interpretative patterns. For further explication of the term see Kimmel 2008 [9] and Sander 1988 [10]. Without an interpretative pattern neither humans nor artificial data processing systems will have a clue what to do with the set of data that is presented to them. If a computer is programmed to interpret a certain type of data, like for instance face-recognition programs, it will not be able to make sense of Chinese symbols. Likewise, a person that has not learned Chinese will neither understand the spoken language nor be able to interpret Chinese symbols, no matter how much sense they can make [11].The systems that may be cognitive, like for instance systems that function according to preset patterns that allow them to analyze data, or even interpret data and transform data into information, are only able to do so within the limitations of its interpretative patterns. If an object recognition program cannot recognize an overturned truck on a highway it is probably because its interpretative patterns (its programming) do not include overturned trucks on highways [12]. If a visually impaired pedestrian is not aware of the signaling system on pedestrian crossings, if the pedestrian lacks an interpretative pattern for that system, and thereby does not know that 3 klicks per second means that they can cross the street $[3 \mathrm{kl} / \mathrm{s}=\mathrm{GO}]$, while 1 $\mathrm{klick} / \mathrm{sec}$ means that they should not $[1 \mathrm{kl} / \mathrm{s}=\mathrm{STOP}]$, the signal is bound to fail in transmitting the information about the status of the traffic on that pedestrian crossing. One could argue that what the pedestrian simply needs is information about the signaling system for pedestrian crossings, not an entire interpretative pattern. Interpretative patterns are like webs of links between informational units that are used by individuals to systematize the interpretation of data. If [3kl/s=GO] were an isolated informational unit not connected to other bits or units of information, it would be applicable in way too many situations (all situations of 3 klicks per second) in which it may be inappropriate to go. In order to apply this correctly, one has to connect to a network of informative bits; traffic-rules, the possible consequences of getting hit by a vehicle at high speed, the position of the source of the klicks in three-dimensional space, parameters of differentiation between types of klicks (if a nervous person klicks a pen nearby or someone sends a message on his smartphone), and many other nodes of informational units that in turn also are connected to other nodes in the network.

Intelligence is often described as connected to information - many times as synonymous to information, as is demonstrated in Nafría et. al. 2016 [13]. Within the context of this analysis, intelligence means to be able to override a pattern and to choose between preset patterns of interpretation in previously not encountered situations. It means being able to investigate - if there is time of course - if a problem is what it looks like at first glance, to be able to question first impressions or the correctness of "given" information. The notion of intelligence is here also related to the notion of information in the sense that a computational act (for instance the process of drawing a conclusion) can be fully rational even though it is based on false information - in which case one can still consider it intelligent. Drawing conclusions, giving feedback in a communicational act, questioning the relevance or truth of a transmitted message, entering 
dialogue, obeying or not obeying a prohibition, are all part of a cognitive system's behavioural pattern. The relation between an interpretative pattern and a behavioural pattern within a system is flexible if the system is intelligent - i.e. if the system is able to adjust behaviour or refuse an impulse to act in a certain way. The concept pair autonomic and automatic are relevant in explaining the functions of the human cognitive system and the outcome of those functions. Also, the connection between autonomic agency and intelligence consists of a cognitive system's being able to reject an input or refuse a command. In Kaber's account (2018) we find the requirements for autonomous agency as follows: "agent viability in a target context, agent self-governance in goal formulation and fulfilment of roles, and independence in defined tasks performance." [14] Action and reaction are central parts of a model of communication (sender, receiver, feedback) and the outcome of the communicational processes are depending on the mechanisms behind the actions and reactions of the communicating agents. Putting automatic before agency seems counterintuitive since the notion of agency is usually connected to matters of choice between courses of action. To define autonomy in such ways that it can be useful for engineering artificial autonomous/intelligent systems, we need to revisit the notion of agency at a later stage when several other connected notions are dealt with.

If knowledge implies (as a minimum) that information is implemented into already existing interpretative patterns, learning must mean to implement new information into already existing interpretative patterns, and occasionally that new interpretative patterns are implemented into the cognitive system. Freire (1968) expresses the relationship between learning and communication like this: "Without dialogue there is no true communication, and without communication there can be no true education." [15]. For Freire, dialogue is simply allowing the student to question and interpret the subject that is being taught before the student implements the message, instead of banking the information into the student - like force-feeding a duck. It means giving the student the possibility to autonomously select the information that is being implemented and to reject implementation if the information is not understood or if the student finds it incorrect or irrelevant. It also means giving the student the possibility of contextualizing the information before the student channels it into appropriate interpretative patterns. Compared to the communication model illustrated by Shannon, Freire gives an indication that the arrows symbolizing transmission of information in the model, especially the arrow that goes from receiver to destination (here baptized "target"), need to be bent or redirected. Even though there are methods to force information upon a target, there is no way of predicting how the target is going to process that information unless some conditions are fulfilled. Without knowledge of the interpretative patterns of the target, the data that is being transmitted (compare to the definition of information above) can be interpreted in many ways, true information can become false and the other way around. Another outcome of forcing information into a cognitive system could be that incompatible "truths" are implemented into the structure and thus cause major defects in behavioural outcome or in computational processes. The incompatibility of truths can be illustrated by relating this discussion to issues of intercultural communication and cross-cultural intelligence testing. Ardilla (2007) emphasises five different cultural aspects that potentially may affect neuropsychological test performance; patterns of abilities, cultural values, familiarity, language and education [16]. An example given by Ardilla, and that occurs quite frequently in intelligence testing of minority children, is that the response to the request of ordering a matrix sequence according to a conceptual principle (the figure that continues the sequence) often is that the teste follows an aesthetic principle (the figure that looks better in that position). All cultural aspects mentioned by Ardilla may affect the interpretative pattern of a person and by that the behavioural outcome following the interpretation. The aspect of natural language and translations of languages is a major obstacle to informational transfer - no matter how accurately translated a message may still be interpreted according to different patterns and result in another informational content than that which is intended by the transmitter. These aspects also affect the natural language processing systems and translation engines that are frequently used to enable 
communication across cultures. They also affect the knowledge content of the education and the informational content in a transmitted message. The accumulation of knowledge in a cognitive system is completely dependent on what type of interpretative pattern that is being employed, and also on the possibility of the learner to autonomously apply interpretative patterns that are appropriate by interacting with the source of information. The target of the transmitted information can, from this point of view, not be regarded as a passive recipient of information, but as an active agent that processes a selected and transmitted set of data into information. In order to be able to determine whether a message that has been transmitted also has "exactly or approximately" reached its destination, the channel between source and destination need to stay open for further informational exchange - or dialogue.

Finally, a concept that is central to describing communication, interpretation, object-recognition, learning, cognition, and other central concepts to our project, is Brentano's (1874) distinction between mental phenomena and physical phenomena. The distinguishing property of the mental from the physical is that of intentionality. Brentano borrows the notion of intentionality from the scholastics and describes it as that feature of the mind that stretches beyond itself and beyond its own content with direction towards an object. This "object" should not be understood as a "thing" or something immanently objective, but rather as a result of an interpretative act, by reaching out to the world intentionality interprets the "something" as something. This intentional act is what gives the object its identity [17]. The informative content of communicative acts concerns human experience of the world, of other people and of self. Natural language is one of the vehicles used to communicate selected bits of information, bits that are not inherently informative but need an interpretative pattern to be turned into information. Both natural language and orientation in and experience of the world/others/self requires extensive use of the human perceptual apparatus. Since every perceptual act requires interpretation by some interpretative pattern for extraction of information (a camera may record visual data but it neither knows that it does so, nor does it have a clue of the informative content of that recorded data), the content of the extracted information may vary from interpretative pattern to interpretative pattern. We aim at resolving this dilemma of quantifying information by modelling the communicational process in such ways that even semantic/meaning content can be quantified - with the aid of the notions we have preliminarily defined above.

Table 1 illustrates the conceptual starting points from which a model of communication that can resolve the meaning/data dilemma can be constructed. We use the labels "necessary" and "sufficient" to mark the criteria we have set for our account of the notions preliminarily defined. In this context, we use "necessary" as a way to delimit the minimal conditions for the concept to carry a certain meaningful content. "Sufficient" is used as a stronger demand, not necessarily as the maximal conditions but as a complement to the necessary conditions that nevertheless do not suffice to make the concept functional in the way we intend to use it in this context. 
International Journal on Information Theory (IJIT) Vol.9, No.3/4, October 2020

Table 1. Explication of central concepts.

\begin{tabular}{|c|c|c|}
\hline Concept & Necessary Criteria & Sufficient Criteria \\
\hline Communication & $\begin{array}{l}\text { an exchange of selected code that is structured } \\
\text { to be interpreted into information }\end{array}$ & $\begin{array}{l}\text { that the selected code structure is } \\
\text { interpreted by an intelligent } \\
\text { cognitive system }\end{array}$ \\
\hline Information & $\begin{array}{l}\text { finite selected sets of data (symbols, code) that } \\
\text { are structured in such ways that they can be } \\
\text { processed into meaningful units by an } \\
\text { independent interpretative pattern }\end{array}$ & interpreted sets of data \\
\hline $\begin{array}{l}\text { Interpretative } \\
\text { Pattern }\end{array}$ & $\begin{array}{l}\text { the cognitive foundation that is systematically } \\
\text { employed by a person at a given point in time } \\
\text { in order to understand a selected sentence or } \\
\text { phenomenon of experience }\end{array}$ & $\begin{array}{l}\text { is composed of patterns of } \\
\text { organization and informational } \\
\text { components, used for object } \\
\text { identification and language } \\
\text { acquisition }\end{array}$ \\
\hline $\begin{array}{l}\text { Behavioural } \\
\text { Pattern }\end{array}$ & $\begin{array}{l}\text { the function generated by the processes } \\
\text { involved in the interpretation of implemented } \\
\text { data as they are interpreted by an agent }\end{array}$ & $\begin{array}{l}\text { flexible, possible to edit by } \\
\text { learning }\end{array}$ \\
\hline Learning & $\begin{array}{c}\text { expanding existing interpretative patterns with } \\
\text { informative content and/or by acquiring new } \\
\text { interpretative patterns }\end{array}$ & entering dialogue \\
\hline Knowledge & $\begin{array}{l}\text { information implemented into an } \\
\text { interpretative pattern structure }\end{array}$ & $\begin{array}{l}\text { the implemented information } \\
\text { corresponds to matters of fact }\end{array}$ \\
\hline Intelligence & $\begin{array}{l}\text { the ability of selecting and processing } \\
\text { information and extracting new information } \\
\text { from a selection of data }\end{array}$ & $\begin{array}{l}\text { exercising the ability of selection } \\
\text { between appropriate interpretative } \\
\text { patterns, also: the ability of } \\
\text { rejecting input and command } \\
\text { autonomously }\end{array}$ \\
\hline Intentionality & $\begin{array}{l}\text { directedness towards an object or } \\
\text { phenomenon }\end{array}$ & $\begin{array}{l}\text { experiencing something as } \\
\text { something }\end{array}$ \\
\hline $\begin{array}{l}\text { Identity } \\
\text { (Objects) }\end{array}$ & $\begin{array}{l}\text { a set of properties grasped by intentional acts } \\
\text { and identified by an interpretative pattern as } \\
\text { belonging to a complex and independent } \\
\text { structure }\end{array}$ & $\begin{array}{l}\text { the composed structure is set as } \\
\text { an object independent of the } \\
\text { intentional acts }\end{array}$ \\
\hline
\end{tabular}

With the aid of these specified concepts we can progress to some ideas on how to quantify the semantic aspect of information. Where Frege's theory of meaning that is based on predicate logic ends - with the question that Frege himself cannot find the answer to - it is necessary to involve the Husserlian theory of perception as the final pieces of the complex process of information extracting and transferring.

\section{QUANTIFYING THE MEANING OF INFORMATION}

As Shannon sets the stage for a mathematical model of communication, he chooses the logarithmic base: "The choice of a logarithmic base corresponds to the choice of a unit for measuring information. If the base 2 is used the resulting units may be called binary digits, or more briefly bits, a word suggested by J. W. Tukey." (1948. p. 379.) A bit is also sometimes called a "shannon" and is the general quantifier of informative content in code. The set of possible messages must be finite though, according to Shannon, so that they can be inserted into logarithmic functions that serve as probability measurements. The point in bringing up Frege (1884) whose development of predicate logic also plays an important part in programming, is that Frege's theory of "bits" of information - as they are described in predicate logic - also are binary, although in Frege's theory the binarity is a marker of meaningfulness. We start off with Frege's account of meaning. 
From a limited set of symbols, like the letters of the alphabet for instance, one can create an unlimited amount of meanings. From letters that form words, and from words that in different combinations form expressions, one can express an infinite set of informational units - if one knows how to compose them. The principle of compositionality states that the meaning of a complex expression comes from its parts. If we flip this coin, we end up with the principle of contextuality which states that the meaning of the parts is composed by the context they are in the expression [18]. To Frege it was important that only complete sentences can be meaningful. A complete sentence is an abstract object that may or may not have a natural language correlate, but the most important aspect, that which makes it complete, it is its structure. Because of their abstract nature, Frege called them "Thoughts", and a complete Thought is a thought that is truthconditioned (can be true or false) [19].We can see the truth-conditions as a test, a way of recognizing a Thought. Recognizing a Thought is similar to recognizing an informative content if it cannot be expressed in a truth-conditional form it is not informative. The structure of a Thought is always composed of a logical subject (a Name as Frege called it), a logical predicate and a relation between them. Expressed commonsensically a complete thought must say something about something, expressed in logical predicative terms "x has the property of being $\mathrm{P}$ " or $[\mathrm{Px}]$.

The Name is not an atomic unit but has its own structure; the something as Something, the question of identity we have mentioned in connection to Brentano's notion of intentionality. What Frege is setting a structure for is informational meaning. The something about Something is the structure of the minimal criteria for informative content - or meaningfulness. A "bit" of information is thus composed by two independent units with different values. "A strawberrish Strawberry" or "A redish Redness" is not informative/meaningful in the sense that "A red Strawberry" is.This is why, besides the structural condition, there has to be a second condition for meaningfulness or informativeness: that the composition is truth conditioned. Tautologies are not informative though. $\mathrm{A}=\mathrm{A}$ and if $\mathrm{A}$ then not not $\mathrm{A}$, are truths that are independent of the matters of the world and human perception.

Tautologies are truth conditioned on the other hand. Truth conditions is a minimal necessary condition for informative content, though not sufficient. A third necessary condition for the sentence to be informative is that it is contextualized and by that truth valued. Another way of saying this is that if the sentence $S$ (that expresses the Thought $[\alpha]$ ) can be valued to 0 or to 1 (is truth-conditioned) then the sentence $S$ is complete though not informative until contextualized. Realizing that in order to determine a truth-value for a truth-conditioned sentence, the sentence has to be contextualized, Frege explains that Thoughts that are being grasped by someone, are contextualized in that grasping and can be truth-valued and as such informative: "One communicates a thought. How does this happen? One brings about changes in the common outside world which, perceived by another person, are supposed to induce him to apprehend a thought and take it to be true." (1956, p 310.) [19].

The sentence 'The strawberry is red.' has the form ' $\mathrm{x}$ is $\mathrm{P}$ ' and is therefore truth-conditioned and also (if true) informative of the state of the world. The words "strawberry" and "red" are in isolation none of the above, neither as Thoughts nor as words. If the strawberry happens to be green, the sentence 'The strawberry is red.' is still informative but not of the state of the world since it is not true. The content though, still makes sense (is informative) since it could be true. This is why Frege did not want the reality of a Thought to be relative to either the world's state of being or the psychological mechanisms that rule human thinking and decision making. Abstract objects are real, and they do have a causal relation to the world in that they affect those who can grasp the Thought and who also act in the world of physical objects. This was Frege's way of saving the notion of meaning from being rendered irrelevant to informative content about reality. 
To summarize the conditions of meaningfulness according to Frege, first there is the binary composition (the something about Something), then we have the measure of rationality provided by truth-conditionality, and finally the principle of contextuality, that which in combination with the other conditions gives a value to the formulation. A problem here is that as soon as we get into truth value issues, we need to involve the human perceptual system to be able to determine the truth of a given structured set of data (a sentence). To Frege the question of how a mind can "grasp a Thought" was still a puzzle that remained to be resolved and which he later attempted to anchor in the use of language as a physiological concrete vehicle of thoughts.

To Frege perceptual experience was not interesting since he classified it as subjective and he needed a concrete/real correlate to the thoughts. Still, Frege had to anchor the thought in a physical reality so that the informative content could be quantified, so the anchorage became - for several reasons - natural language. To Frege the Husserlian notion of meaning was leaning towards psychologism, to which Husserl replied that it is not necessarily so [20]. Some Thoughts may be irrational (according to predicate logic structures) and therefore only meaningful to those who grasp the Thought. Irrational Thoughts can seldom be successfully communicated and very often they do not have any informative content at all. As such, irrational thoughts, in spite of being meaningful to irrational cognitive structures, have no informative value and lack a general meaning that can be transferred to other cognitive structures.

What we have gained by the reality of Thoughts and the Principle of Contextuality for the context of communication theory, is a distinction that can be translated (for the purposes of context) to the distinction between data and information. Data, in contrast to information, can be controlled and is easy to quantify, information is the product of an individual's interaction with data. It is the grasped Thought that can produce knowledge (information), so the information-symbol on a model of communication must always be attached to an agent that can grasp the Thought (see Figure 3. below). But since the term Thought so easily can be interpreted in the psychological sense that Frege tried to explain away from the concept, this terminology is not practical to use it in the context of communication theory. Instead, I will use "bit" or "data" or "informative content" to refer to the binary units that are truth-conditional but not truth-valued, and "information", "meaning", "value" for the units that are contextualized data - Thoughts that have been grasped. So, to let Frege's question point us ahead, exactly how is a Thought grasped?

\section{IDENTIFICATION AND RECOGNITION OF OBJECTS}

Husserl distinguished between sense-data (hyle) and intentional meaning (morphe) [21]. In Husserl's theory of perception, it is raw data (hyletic data) that is within interpretational range that gets interpreted in the acts of perception. In the terminology used in this paper this would translate to; intentionality is that which makes sense of data and transforms it (or translates it) into information. Intentionality functions as that which reaches out to whatever is within interpretational range, a process initiated by selection of available content. Constitution of phenomenal objects (the objects of perception) is a process that requires more than a passive receptive standing; listening, seeing, smelling and feeling, all require an activity that here is termed interpretation. Interpretation is a complex process that involves a large number of faculties and is underlying many, if not all, the cognitive functions in a cognitive system. They cannot all be accounted for within this context.

What it basically means to perceive in this theory, is that in the act of interpretation the hyletic data are organized, contextualized and structured into meaningful objects. The meaning of the sounds that I interpret as a car passing by is obviously not inherent in the generated sound waves themselves, I have to compose their meaning by contextualizing them, using my interpretative patterns to do so. This means that the world does not enter our cognitive system through the 
senses in organized form (like the models of communication suggest), but in much more chaotic ways for which we need our interpretative faculties to make sense of them. At this point comparisons with object-detection programming may reveal some operational differences and similarities. After all, it is the human perceptual system that stands as a model for artificially constructed cognitive systems. The areas in which object-recognition in artificial systems remains challenging are often related to a function in humans that is not always accounted for even in mappings of the human cognitive system: apperceptions.

The interpretative faculty not only composes and organizes the hyletic data into meaningful objects, it also fills in the blanks with the assistance of already acquired informative content that is implemented in the cognitive system's interpretative patterns. Husserl calls these fillings apperceptions. Raw data, or hyletic material, are merely Abschattungen in Husserl's terminology, "shadows" of the reality behind our perceptions. By a change of perspective, or merely the passing of time, the visual datum changes continuously, it is time-space dependent. A good illustration of this function is given by the studies of how blind-born learn to "see" after eyesurgery that restores their sight. Constantly changing colour-patches do not help distinguish between objects in the environment, visual data require continuous interaction with the surroundings, an interaction in which different kinds of raw input can be structured and composed into the objects that deserve our attention. To actually identify objects requires a number of functions, and among these apperceptions are strictly necessary. The function that apperception has on the perceptive system may be illustrated by the image below:

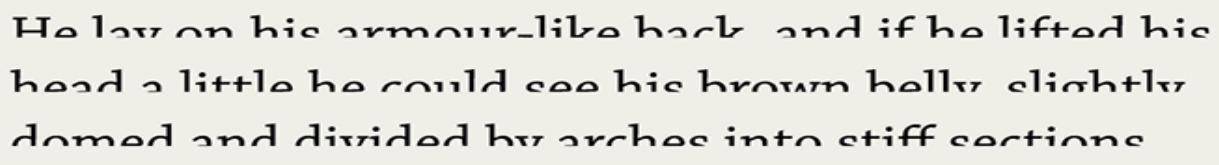

Figure 2. Missing data. [22]

Even though the data in the image above is severely damaged, most possessors of the interpretative pattern of the English language can easily read the text and extract a number of informative bits. The point here is that damage to the code does not imply damage to the informational content. Also, the meaning extracted by English readers is mainly on a basic level unless one can identify the source of the original text. There seems to be no upper limit to how many levels of meaning one can add to a selection of code, but this is not an obstacle to quantification. The important feature for communication is the lower limit at which humans can discern patterns and decipher them into meaningful informative units.

Another feature of perception that Husserl draws our attention to is the setting of existence. We objectify the structures that we have composed with our intentional acts, we set an existential quantifier in front of it (existence is not observable, but yet another apperception that is included in the act of constituting an object), we experience those objects as real (meaning they have an identity independent of our experience of them) and we identify them as objects or properties of objects. We even give them Names so that we can communicate our experience of these objects to others, and so that we can make sure that we are talking about (exactly or approximately?) the same thing. We need to do this existential setting, since we might have to interact with or react to the physical reality around us. This feature is not implemented in any known artificial cognitive system, but if knowledge, observations and communication require not only data but also action and interaction of some sort, it is a feature worth considering for development of intelligent cognitive systems. What type of agency is required for object identification and object recognition? 
Action and interaction require some form of agency. We initially separate between two main forms of agency: automated and autonomous. As much as we have been inspired by Kaber's (2018) attempt to differentiate between autonomous and automated agents, we still see the possibility of designing systems that are automatic with elements of autonomy - just as we can identify moments of automated agency and moments of autonomic agency in the one and same individual. Kaber also defines 'agent' as "a 'thing' in an environment with capacities to sense states and effect aspects of the environment" [14]. Agency on the other hand, is in our account an ability to interact with the environment, whether the action is supported by pre-set (mechanic) processes or a result of decisions made by a sensate agent. As Linell (2016) suggests, autonomous agency is involved in the making of meanings in communicative acts [23].

Following Kaber's account of autonomous agents, the sense of goal-directedness is adopted and, just like intentionality, it is here considered as a main component of an intelligent cognitive systems perceptual faculty. One interesting feature that we have attempted to ascribe to intelligence, is the ability to override a command (supress automated reactions) or to question the truth/understanding of a proposition. Linell ascribes this feature to sensitive agency: "Note that agency, on this analysis, includes the ability to suppress impromptu reactions, something which might look like nonaction. Somebody who cannot keep his/her impulses and emotions in check, can be held accountable for ignoring or neglecting such a possibility. Also, sensitive agency is related to being a good listener, acting as somebody who has respect for the other's need to have time (now and then) for thinking during interaction. "(2016, p. 40.) It is necessary to relate goaldirectedness to both intentionality (as it has been explicated above) and to object-recognition processes. The object-recognition process is in humans, as Husserl's theory of perception illustrates, reliant on intentionality, apperception and the intentional acts as constructors of objects.

\section{The Realist Model of Communication}

One of the purposes of a model of communication has in this paper been identified as predictability. Predictability was also the reason that the designers of the original models of communication gave for not taking into account the semantic aspects of information in communicational processes. Here, we have made an attempt to recognize the role of the semantic aspects as necessary to the notion of information without neglecting the aspect of predictability, by introducing a way of quantifying the semantic aspects of informational content. Without the aspect of interpretation, it is not possible to enter a communicational act, a dialogue, with the object or person that figures as a source of information. Without dialogue it is not possible to evaluate the success of the informational transaction. Without the rationality aspect, on the other hand, the request for structure for the possibility of interpretation, it is also not possible to evaluate the success of an informational transaction. Mainly dialogue does not assure that we are talking about the same thing or that we are making sense at all. Also, the semantic aspect does not have to be reduced to statistical probability outcome, and we do not have to sacrifice the aspect of interpretation to account for a successful informational transfer. By this we also argue that including the semantic aspect in information we need not lose any of the expected predictability.

In short; a selected set of structured data (for the structure we have Frege's necessary conditions: binarity, truth-conditionality and context) needs to be interpreted into informative content by an agent that (either automatically or autonomously) makes use of the interpretative patterns it possesses. In case of lack of interpretative pattern for that specific set of structured data (like a foreign natural language), the agent must choose between acquiring a new interpretative pattern as an aid to new acts of interpretation, or, consider the set as meaningless and not informative. The process of interpretation is involved at all levels of the extraction of information. The first 
step is thus to identify or recognize what type of interpretative pattern that is needed for the selected set of data. (Inappropriate interpretative pattern will either distort the interpretation of data or not reveal all of its contents. Compare to intercultural communicational situations discussed in section 2.) When the informative content has been extracted it needs to be presented in the appropriate way to make it available to the agents that are targeted as receivers of the informative content (like for instance translation between natural languages or adaption of the form of presentation to the target's known interpretative patterns). For the informative content to be communicated to a target or targets, the agency of the target is required just as much as the agency of the agent selecting and structuring the set into appropriate form. The target for the presentation needs to activate the same interpretative functions that the initial selector and extractor of informative content has employed. The original selector may not have constructed the contents properly for the target to grasp, so to be able to make sure that the message has been reproduced "exactly or approximately" as it has been selected, the target needs to have the possibility of dialogue. The possibility of asking questions to the selector of content is part of the process of interpretation. If a content gets implemented in a cognitive systems interpretative patterns (the agent learns) without being able to make sure that the content is interpreted in accordance with the original selection, or even without being able to make sure that the content is represents some matters of fact or what type of matters of fact it represents, that content may cause damage to the entire interpretative pattern and collapse the whole communicative process.

We can now identify three different processes involved in a communicational act; interpretation (which includes the selective process, contextualization, and dialogue), presentation (recontextualization, making accessible to target) and implementation (learning). The components that are necessary to illustrate the functions that these processes are functional to are; agent(s), interpretative patterns (and behavioural patterns, to which we will return in sections ahead), code and information. Different communicational situations may involve only some of the components or processes, though it is impossible - according to this model and the definitions we have formulated earlier - to involve any information without the process of interpretation. An illustration of the connections just described could be represented in the following form:

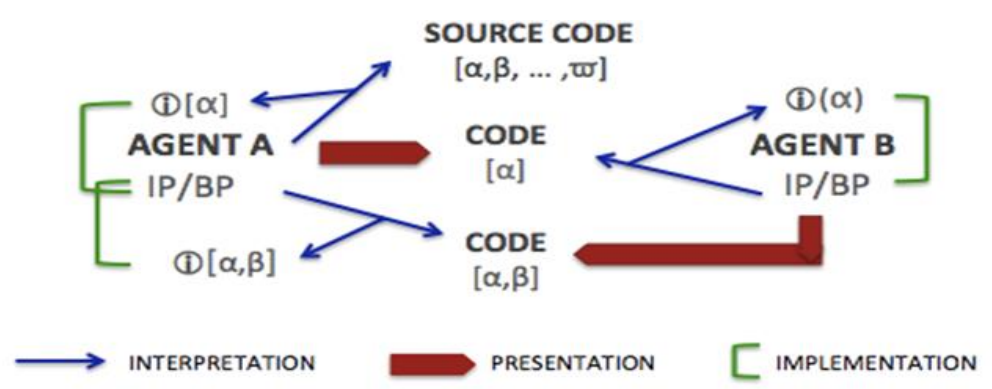

Figure 3. A realist model of communication

Agent A selects a structured set (from a source), interprets the structure into meaningful informative bits, presents it in an appropriate way depending on the communicational situation, and Agent B interprets the selection into information. Note that it is not in information-form that the informative content is presented. The act of presentation can be viewed as a form of translation to whatever code that the target for the informative content is assumed to be able to decode/interpret. The information is only at the point of the interpreter.

In some communicational situations it is not necessary that the interpreted information be implemented. Some situations do not even require the interpretation of the content, selected sets of data can be transmitted without the transmitter's knowing about the informational content. The presentation in those situations becomes impossible to control or to adapt to the target by the 
transmitter. It also becomes very difficult for the target, Agent B, to enter a dialogue with the transmitter so that Agent B can make sure that his or her interpretation of the selection is correct, or if it correlates to some matters of fact, or to contextualize the information (which also is an impediment to the act of interpretation). When it comes to implementing the informational content into already existing patterns of interpretation (that which in popular terms is called 'learning'), most people find it practical to not implement false informative content. Informative content tends to have an impact on the actions of individuals, an effect which is discussed at length in connection to our examination of behavioural patterns, and if the informative content does not correspond to any matters of fact or is not properly contextualized, the effects may be severely affecting the possibility of damage control in behavioural output (an agent may still be rational even if the informative content behind the action is incorrect). The challenge that the spreading of disinformation poses is also a well-known factor in international conflict situations.

We consider this model of communication to be general, in the sense that it can be applied to all types of situations where informational transfer is involved, and to all types of agents and agencies. It can even be applied on all levels of human activity - from a researcher's attempt to expand knowledge in a field and then communicate this knowledge to others, to a person's safely crossing the street.

This being a model for informational transfer (communication) it may not be applicable to informational technology. Informational technology is not constructed to perceive, or intentionally direct itself towards a world independent of its own being, or to question/refuse the input/command that enters its personal space. A data-processing machine can therefore not be said to process information or transmit information. This implies a number of limitations to the operations it can perform. This also implies it cannot think, but more importantly, it will also come to the point where it can no longer imitate the ability of thinking in a satisfactory way. The dangers of thinking machines is a subject matter for a different context. What can be said here, is that human communication is necessarily dependent on the functions described in this paper, and therefore, if the purpose of informational technology and of artificial cognitive systems is to mimic human thinking and behaviour (even in ways that exceed human capacities), it is necessary to construct machines that process information - not data.

The alternative is to limit machine functionality to processing data and leave the task of processing and managing information to humans. If we were to perform an operation on the Shannon model, where we disconnect the source and the destination nodes and connect the body of the model to the realist model, we might end up with a model that can be applied to all communicational situations, including those that are channelled through informational technology.

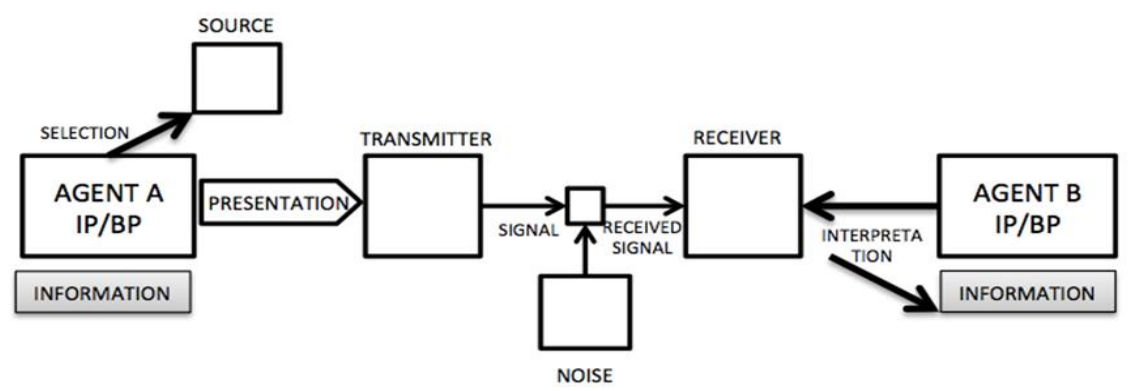

Figure 4. Simplified combinational version. 
In some communicational situations there is a need for a translator or interpreter. In those cases, the easiest way to connect the translator is to add an Agent $\mathrm{C}$ to the realist model. The realist model can be modified to any communicational situation as long as the processes involved in the informational transfer are represented correctly and the resulting information is positioned properly in accordance with the realist theory of information. The realist model does not exclude the computational model; in some communicational situations the computational model is necessary to include in the process of informational transfer. Though it should not carry the whole burden of transmitting information until it is suitable for representing informational processing.

\section{The WAY AHEAD}

Setting out to resolve the engineering problem as it was formulated by Shannon in 1948 is probably one of the most challenging tasks in information theory today. Starting by questioning the applicability of the concept of information that has prevailed since 1927, when Hartley separates the physical and psychological aspects of information in his attempt to quantify information, the argument in this paper has been along the lines that a notion of information that does not take the interpretative aspects into consideration is incapable of functioning as a foundation for informational transfer. This feature has not only been an impediment in various communicational situations, it has also led to several limitations for the development of assistive technology and artificial systems that process language, recognize objects and learn. Through an attempt to find a basis for quantification of the quality of information - the aspect of meaning by interpretation - the author presents a flexible model of communication that can be adjusted to most, if not all, communicational situations.

The ability to interpret data into informative units is a feature that we have not yet fully disclosed in all its elements and functional aspects. What we have to look forward to is the discovery of how applications of the realist model of communication to different types of communicational situations can improve success in informational transfer. This of course includes informational transfer through informational technology. There is more work to be done on both the theoretical and the applicational levels, but resolving the remaining challenges is a pleasant task that we can look forward to if we take into consideration the benefits that may result from that work with respect to successful informational transfer.

\section{REFERENCES}

[1] Shannon, C. E. (1948): "A Mathematical Theory of Communication" in The Bell System Technical Journal 27, July 1948, pp 379-423.

[2] Hartley, R. V. L. (1927): "Transmission of information", paper presented at the International Congress of Telegraphy and Telephony, Lake Como, Italy, September 1927. Copyrighted by Lucent Technologies, 1928.

[3] Weaver, W. (1949): "The mathematics of communication" in Scientific American, vol. 181, no 1, July 1949, pp 11-15.

[4] Bao, J. et. al. (2011): "Towards a theory of semantic communication. (Extended Technical Report)", Performing Organization: US Army Research Lab, Adelphy, Presented at the 2011 IEEE First International Workshop on Network Science.

[5] Kimmel, M., Mutto, M. (2019): "Report: An analysis of communication models and their applications since 1948", Copyrighted by Collibri Text 2019. Open source: https://collibri.se/collibri-text.

[6] Turing, A. M. (1950): "Computing machinery and intelligence", Mind 49: 433-460.

[7] Capurro, R. and Hjørland, B. (2004): "The concept of information", in Annual Review of Information Science and Technology, Theorising Information and Information Use, Section IV, Chapter 8, 2004. 
International Journal on Information Theory (IJIT) Vol.9, No.3/4, October 2020

[8] Bawden, D. and Robinson, L. (2014): "'A few exciting words': Information and entropy revisited", Journal of the Association for Information Science and Technology 2014, published online in Wiley Online Library (wileyonlinelibrary.com). DOI: 10.1002/asi.23459.

[9] Kimmel, M. (2008): Interpreting Mysticism. An evaluation of Steven T. Katz's argument against a common core in mysticism and mystical experience.VDM Dr. Müller Verlag 2010. University of Gothenburg edition: 2008.

[10] Sander, Å. (1998): En tro - en livsvärld. En fenomenologisk undersökning av religiös erfarenhet, religiöst medvetande och dess roller i livsvärldskonstitutionen, Filosofiska Meddelanden, Röda Serien, 1988.

[11] Searle, J. (1980): "Minds, Brains, and Programs", in Behavioural and Brain Sciences 3, 417-424.

[12] Tempelton, B. (2020): "Tesla in Taiwan crashes directly into overturned truck, ignores pedestrian, with autopilot on.", Forbes, Editors pic, June 2, 2020. https://www.forbes.com/sites/bradtempleton/2020/06/02/tesla-in-taiwan-crashes-directly-intooverturned-truck-ignores-pedestrian-with-autopilot-on/\#469f58458e56

[13] Nafría, J. M. D. et. al. (2016): Interdisciplinary Elucidation of Concepts, Metaphors, Theories and Problems Concerning INFORMATION, GlossariumBITri, 2016.

[14] Kaber, D. B. (2018): "A conceptual framework of autonomous and automated agents", in Theoretical Issues in Ergonomics Science, Volume 19, 2018, Issue 4, pp 406-430.DOI: 10.1080/1463922X.2017.1363314

[15] Freire, P. (1968): Pedagogy of the oppressed, New York: Continuum 2007.

[16] Ardilla, A. (2007): "The impact of culture on neuropsychological test performance", in International Handbook of Cross-Cultural Neuropsychology, Lawrence Erlbaum Associates 2007, pp 23-44.

[17] Brentano, F. (1995): Psychology from an empirical standpoint, Routledge 1995. First published in 1874.

[18] Frege, G. (1884): The foundations of arithmetics. A logico-mathematical enquiry into the concept of number. First Harper Torchbook, New York 1960. First published 1884 with the title Die Grundlagen der Aritmetik.

[19] Frege, G. (1918): "The thought. A logical inquiry." Mind, New Series, Vol. 65, No. 259 (July 1956), pp. 289-311. First published in 1918 in Beiträgezur Philosophie des DeutschenIdealismus, 1918-

[20] Beyer, C. (2017): "Husserl and Frege on Sense", in Centrone S. (ed.) Essays on Husserl's Logic and Philosophy of Mathematics, Springer Verlag (2017).

[21] Husserl, E. (1913): Ideen zu einerreinenPhänomenologieundphänomenologischenPhilosophie, Max NiemeyerVerlag Tübingen, 1993. First published in 1913.

[22] Kafka, F. (1915): "Metamorphosis", first published 1915 by Kurt Wolff Verlag.

[23] Linell, P (2016): "On agency in situated languaging: Participatory agency and competing approaches.", New Ideas in Psychology 42 (2016) 39-45. 